\title{
Einsatz eines Wendelappens zur Wiederherstellung des Volumens im Achselbereich - ein neuer Behandlungsansatz für die operative Versorgung von sekundären Lymphödemen nach Brustkrebstherapie Re-augmentation of the Axilla Using a Turn-Over Flap - a New Approach for the Surgical Treatment of Secondary Lymphedema After Breast Cancer
}

\author{
Autoren

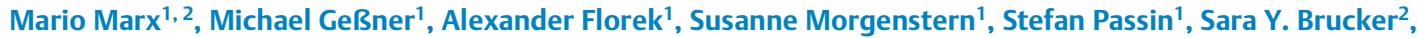 \\ Diethelm Wallwiener², Markus Hahn²
}

Institute

1 Elblandklinikum Radebeul, Klinik für Plastische, Rekonstruktive und Brustchirurgie, Radebeul, Deutschland

2 Universitätsklinikum Tübingen, Department für Frauengesundheit, Tübingen, Deutschland

Schlüsselwörter

Lymphödem, Mammakarzinom, Lymphknotentransplantation, Achsellymphknoten, brustkrebsassoziiertes Lymphödem

Key words

lymphedema, breast cancer, lymph node transplantation, axillary node, breast cancer-related lymphedema

eingereicht 13.09.2019

akzeptiert 18.11.2019

\section{Bibliografie}

Senologie 2021; 18: 266-272

DOI $10.1055 / \mathrm{a}-1441-2575$

ISSN 1611-6453

(c) 2020. The Author(s).

This article was originally published by Thieme in Geburtsh Frauenheilk 2020; 80: 526-531 as an open access article under the terms of the Creative Commons Attribution-NonDerivative-NonCommercial License, permitting copying and reproduction so long as the original work is given appropriate credit. Contents may not be used for commecial purposes, or adapted, remixed, transformed or built upon. (https://creativecommons.org/licenses/by-nc-nd/4.0/)

Georg Thieme Verlag KG, Rüdigerstraße 14,

70469 Stuttgart, Germany

Zitierweise für diesen Artikel Geburtsh Frauenheilk 2020; 80: 526-531. doi:10.1055/a-1063-6310

Korrespondenzadresse

Prof. Dr. med. Markus Hahn

Universitätsklinikum Tübingen

Department für Frauengesundheit, Calwer Straße 7,

72076 Tübingen, Deutschland

markus.hahn@med.uni-tuebingen.de

\section{ZUSAMMENFASSUNG}

Einleitung Narbige Verengungen und ein Volumendefizit im Bereich der Axilla können zu einem anhaltenden Lymphödem führen und zählen zu den häufigsten Nebenwirkungen nach einer Brustkrebstherapie im Achselbereich. Ziel dieser Arbeit ist die Vorstellung einer Operationstechnik zur Reduzierung von Lymphödemen mithilfe eines Turn-Over Flaps.

Material und Methoden Zwischen Oktober 2016 und Mai 2018 wurden 5 Patientinnen mit einem Lymphödem Grad 2 nach Mammakarzinomtherapie mit dieser neuen Methode behandelt. Dabei erfolgte die vollständige Entfernung der kompromittierenden Narbenstränge der Axilla, gefolgt von einer Reaugmentation der Axilla mittels Turn-Over Flap. Danach wurde bei allen Patientinnen eine Brustrekonstruktion mit einer an die thorakodorsalen Gefäße angeschlossenen Fernlappenplastik durchgeführt. Prä- und postoperativ wurden der Umfang beider Arme, die Lebensqualität und die Schmerzen gemessen.

Ergebnisse Im Durchschnitt sanken die Schmerzen im ipsilateralen Arm von 6 auf 1 auf der numerischen Schmerzskala, und die Lebensqualität verbesserte sich merklich $(2,8$ vs. 7,0$)$. Es zeigte sich eine deutliche Verringerung des Lymphödems, vor allem im Oberarm. Komplikationen wie sekundäre Blutungen, Infektionen oder Lappenverlust sind nicht aufgetreten. Schlussfolgerung Eine vollständige Entfernung der nach Brustkrebsbehandlungen entstandenen Vernarbungen im Bereich der Axilla und eine Reaugmentation der Axilla mittels Turn-Over Flap führten zu einer Verringerung des ipsilateralen Lymphödems und zu einer Verbesserung der Schmerzen und der Lebensqualität. Wir deuten dieses Outcome als Ergebnis der physiologischen Ausrichtung der Lymphgefäße und eines lymphknotenhaltigen Transpositionslappens. Die Reaugmentation der Axilla mittels Turn-Over Flap könnte eine wirksame, nicht mikrochirurgische Alternative zur Lymphknotentransplantation darstellen.

\section{ABSTRACT}

Introduction Scarring and volume reduction of the axillary space resulting in persistent lymphedema is a side effect of axillary treatment in breast cancer patients. The aim of this 
paper is to describe the reduction of lymphedema achieved with a turnover flap.

Materials and Methods Between October 2016 and May 2018, five patients with grade 2 lymphedema following breast cancer therapy underwent reconstructive surgery with a turnover flap. Complete excision of the symptomatic axillary scar tissue followed by re-augmentation using a turnover flap was performed. Subsequently, all patients underwent breast reconstruction using a distant flap reconnected to the thoracodorsal vessels. The circumference of both arms, quality of life and pain were measured before and after surgery.

Results An average reduction in pain in the ipsilateral arm from 6 to 1 on the numerical scale as well as an improvement in quality of life (2.8 vs. 7.0) was observed. A decrease in lymphedema especially in the upper arm was identified. No complications such as secondary bleeding, infections or flap loss were observed.

Conclusion Complete removal of the axillary scar tissue after breast cancer treatment and re-augmentation of the axilla with a turnover flap results in a reduction in ipsilateral lymphedema and an improvement in pain and quality of life. We interpret these findings as a result of the physiological re-alignment of the lymphatic vessels combined with a lymph node containing local transposition flap. Therefore, re-augmentation with a turnover flap could be an effective nonmicrosurgical alternative to lymph node transplantation.

\section{Einleitung}

Eine der Hauptkomplikationen nach operativer Behandlung und Strahlentherapie des Mammakarzinoms ist das Lymphödem der oberen Extremität [1]. Klinische Erfahrungen und aktuelle Studien belegen, dass unter den jährlich 69000 Patientinnen in Deutschland mit primärem Mammakarzinom die Häufigkeit des therapiepflichtigen Lymphödems bei 16,6\% liegt und dieses somit etwa 11500 Patientinnen betrifft [2]. Diese Störung des Lymphabflusses kann die Lebensqualität erheblich einschränken und ist vor allem Folge der Lymphadenektomie und/oder Strahlentherapie [2, 3]. Die Inzidenz des Lymphödems 12-24 Monate nach einer Brustkrebsbehandlung mit Ausräumung der axillären Lymphknoten liegt bei 19,9\% [2]. Patienten, die sich einer Axillaausräumung und Bestrahlung unterzogen haben, weisen ein noch höheres Risiko auf, ein Lymphödem zu entwickeln [3-5]. Nach Einführung der Sentinel-Lymphknoten-Biopsie beim Mammakarzinom sank das erwartete Risiko auf 6-10\% [7]. Komplikationen sind jedoch nicht nur Folge behandlungsbedingter Risikofaktoren, sondern auch durch patientenbezogene Faktoren, beispielsweise Adipositas und Hypertonie, bedingt [5, 6, 8, 9]. Unbehandelt führt das Lymphödem oft zu einem progredienten Krankheitsverlauf mit zunehmender Symptomatik. Da die rekonstruktive Chirurgie bei den meisten Patienten/-innen die operative Therapie der ersten Wahl ist, hat es gerade auf diesem Gebiet zahlreiche Fortschritte gegeben. Baumeister führte 1981 die erste mikrochirurgische Lymphgefäßtransplantation durch [10]. Corinne Becker veröffentlichte im Jahr 2000 den ersten Bericht über die freie Lymphknotentransplantation [11]. Die aktuelle deutsche Leitlinie befürwortet die Lymphgefäßrekonstruktion bei Patienten/-innen mit progredienter Erkrankung, bei denen die konservativen Therapieoptionen ausgeschöpft sind [12].

Basierend auf der Erfahrung von über 2000 Brustrekonstruktionen mit einer freien Lappentechnik und Anschluss an die Vasa thoracodorsalia haben wir an den ipsilateralen oberen Extremitäten einen erstaunlichen Effekt festgestellt. Viele Patientinnen berichteten über einen Rückgang des ipsilateralen Lymphödems. Der gleiche Effekt stellte sich nach operativer und strahlentherapeutischer Behandlung des Mammakarzinoms mit symptomatischer axillärer Narbenbildung ein, als das Narbengewebe entfernt wurde. Zur Prophylaxe des Narbenrezidivs bauen wir den entstandenen Gewebedefekt mit einem lokalen Lappen wieder auf. Die vollständige Exzision des Narbengewebes mit anschließender Wiederherstellung des Volumens hatte also einen Effekt, den wir als Folge einer Neuausrichtung der chronisch abgeknickten Lymphgefäße ( $\triangleright$ Abb. 1) und des Einschwenkens eines lokalen Lappens mit Lymphknoten (Turnover-Flap) werten. Ziel dieser Arbeit ist es, anhand von 5 repräsentativen Fällen diese Operationstechnik und den erreichten Rückgang des symptomatischen Lymphödems nach vollständiger Resektion des axillären Narbengewebes und anschließender Wiederherstellung des Volumens mit einem Turn-Over Flaps zu beschreiben.

\section{Material und Methoden}

Diese retrospektive Betrachtung bezieht sich auf 5 Patientinnen, die sich nach Mastektomie mit einem trotz konservativer Behandlung persistierenden Lymphödem Grad 2 vorstellten und sich einer Brustrekonstruktion mit gleichzeitiger kompletter Resektion des Narbengewebes und Wiederaufbau der Axilla mittels TurnOver Flaps ( $\triangleright$ Tab. 1) unterzogen. Sämtliche Daten in dieser retrospektiven Auswertung wurden anonymisiert. Die zuständige Ethikkommission wurde über die retrospektive Studie informiert. Alle Patientinnen gaben ihr schriftliches Einverständnis. Vor dem Eingriff wurden die Patientinnen mindestens 6 Monate lang konservativ behandelt, was entweder zu keiner Verbesserung bzw. sogar zu einer Verschlechterung des Lymphödems führte. Zusätzlich zum Wiederaufbau der Axilla mit einem Turn-Over-Flap planten wir eine Brustrekonstruktion mit perforatorbasiertem freiem Lappen. Die Patientinnen wurden prä- und postoperativ fotodokumentiert ( $\downarrow$ Abb. 2 ), es erfolgten standardisierte Umfangsmessungen an beiden Armen, und die Patientinnen beantworteten einen validierten Fragebogen zur Lebensqualität bei Lymphödem (LYMQOL) [13]. Die Bewertung basierte auf 20 Fragen in 4 Domänen: Funktion (Q1-3), Aussehen (Q4-8), Symptome (Q9-14) und Stimmung (Q15-20). Die Bewertung der einzelnen Items reichte von 1 (überhaupt nicht) bis 4 (sehr viel). Die Schmerzanamnese wurde anhand einer numerischen Ratingskala von 0 (keine Schmerzen) bis 10 (stärkste Schmerzen) festgehalten. Nachuntersuchungen fanden in Woche 1 und 6 sowie 6 Monate nach dem 


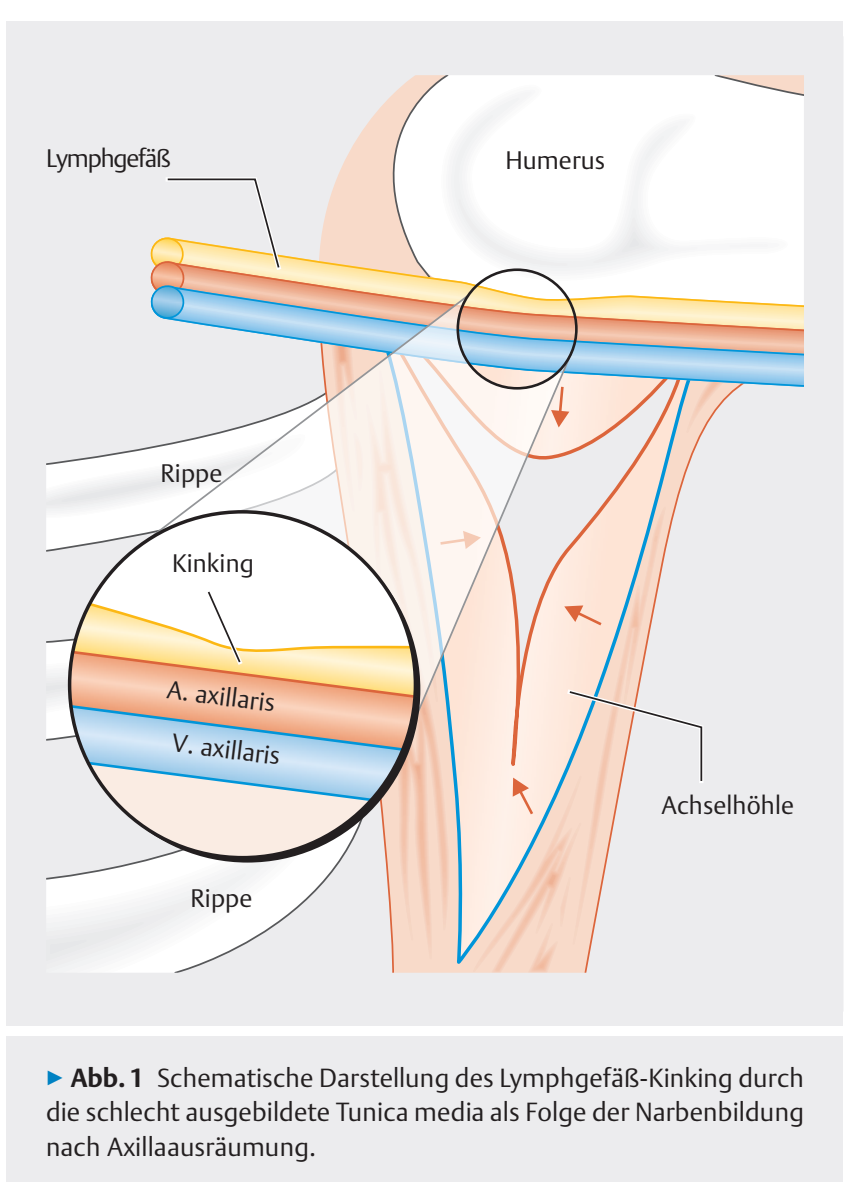

Eingriff statt. Zur Vermeidung von tageszeitlichen Schwankungen des Lymphödems erfolgten die Messungen immer zur gleichen Tageszeit ( \pm 1 Stunde).

\section{Operationsmethode}

Zentraler Pfeiler dieser Technik ist die vollständige Resektion des durch vorherige Operationen und Strahlentherapie in der Achselhöhle entstandenen Narbengewebes. Bei vollständiger Ausräumung des problematischen Narbengewebes kommt es in der Axilla zu einem Volumendefekt. Die Vasa thoracodorsalia et thoracolateralia und die Nerven werden bei der Präparation vollständig dargestellt und dienen dem Anschluss des Perforatorlappens. Die Darstellung $\operatorname{der} A$. et $V$. thoracica lateralis mit anhaftendem lymphknotenhaltigem Fettgewebe distal der V. axillaris am lateralen Rand des M. pectoralis major erfolgt mittels intraoperativen Ultraschalls (12 MHz, B-Mode und Farbdoppler) und Lupenbrille. Das Lymphknoten-Fettkonglomerat sollte so groß sein, dass es den vorhandenen Volumendefekt ausfüllt ( $\mathbf{A}$ Ab. 3). Der adipofasziale Insellappen wird dann zusammen mit der Faszie des M. serratus anterior distal inzidiert. Nach Clipverschluss der Gefäße distal des Turn-Over Flaps wird das Lymphknoten-Fett-Konglomerat durch den von der Narbengeweberesektion geschaffenen Raum zur V. axillaris gedreht. Der zuvor entstandene Gewebedefekt wird so durch ausreichend vaskularisiertes Lymph- und Fettgewebe aufgefüllt. Anschließend wurde eine autologe Brust- rekonstruktion mit einem DIEP-Lappen (deep inferior epigastric perforator) durchgeführt ( $\triangleright$ Abb.4).

\section{Ergebnisse}

Eine Reaugmentation mittels Turn-Over Flap mit vollständiger Nachkontrolle erfolgte bei 5 Patientinnen ( $\triangleright$ Tab. 1).

Alle Patientinnen wiesen präoperativ ein Lymphödem zweiten Grades auf, mit schmerzhaften Bewegungseinschränkungen infolge einer Umfangsvermehrung des betroffenen Armes und einer damit einhergehenden Einschränkung ihrer Lebensqualität. Alle Patientinnen hatten vor dem Eingriff eine mindestens 6-monatige konservative Behandlung durchlaufen, worunter die Symptomatik sich jedoch weiter verschlechtert hatte. Von 3 Patientinnen lagen sämtliche Messungen und Fragebogen vor. - Abb. 5 zeigt das vorliegende Lymphgewebe und die Durchblutung, die durch präoperative Injektion von Indocyaningrün in die seitliche Brustwand und die Hand der Patientin mittels einer Infrarotkamera sichtbar gemacht wurden.

\section{Schmerz}

Das von den Patienten auf einer numerischen Analogskala angegebene mittlere präoperative Schmerzniveau lag bei 6 (2-8). Sechs Wochen nach dem Eingriff berichteten alle Patienten über einen Rückgang der Schmerzen in der betroffenen Extremität. Der mittlere Schmerzscore lag bei $1(0-3)$, wobei 2 Patientinnen angaben, völlig schmerzfrei zu sein.

\section{Konservative Therapie}

Sechs Wochen nach dem Eingriff benötigten 3 der 5 Patientinnen keine weitere konservative Behandlung des Lymphödems. Die beiden anderen Patientinnen konnten die Abstände zwischen ihren konservativen Behandlungssitzungen vergrößern. Keine der Patientinnen musste eine Kompressionsmanschette tragen.

\section{Umfang des betroffenen Arms}

Die Messungen am Arm erfolgten am Handgelenk, in der Ellenbeuge sowie $5 \mathrm{~cm}$ proximal und distal des Ellenbogens und am Oberarm nahe der Axilla. Der stärkste Rückgang des Lymphödemumfangs nach 6 Wochen zeigte sich am Oberarm mit einer mittleren Besserung um 3,57 cm vs. 1,1 cm im kontralateralen Arm, gefolgt von der Lokalisation $5 \mathrm{~cm}$ proximal der Ellenbeuge $(1,1 \mathrm{~cm}$ vs. $1,5 \mathrm{~cm})$ und am Handgelenk $(1,0 \mathrm{~cm}$ vs. $0,6 \mathrm{~cm})$.

\section{Lebensqualität}

Postoperativ besserte sich die Lebensqualität insgesamt um 4,2 Punkte (2,8 vs. 7) auf einer numerischen Analogskala.

Der LYMQOL-Fragebogen zur Lebensqualität dokumentierte ebenfalls eine Besserung des Lymphödems. Der präoperative Score unserer Patientinnen lag im Mittel bei 59,2 (47-74). Postoperativ sank der mittlere Schmerzscore um 13,6 Punkte auf 45,6 (30-64) und entsprach damit ebenfalls einer Besserung der Lebensqualität. 
\ Tab. 1 Zusammenfassung der demografischen Daten unserer 5 Patientinnen sowie Auswertung der prä- und postoperativen Scores für Schmerz und Lebensqualität.

\begin{tabular}{|c|c|c|c|c|c|c|}
\hline \multicolumn{2}{|l|}{ Patientin } & 1 & 2 & 3 & 4 & 5 \\
\hline \multicolumn{2}{|l|}{ Alter } & 59 & 50 & 43 & 59 & 48 \\
\hline \multicolumn{2}{|l|}{ BMI $\left(\mathrm{kg} / \mathrm{m}^{2}\right)$} & 24,7 & 36,5 & 25,9 & 31,8 & 24,22 \\
\hline \multicolumn{2}{|l|}{ Grad des Lymphödems } & Grad 2 & Grad 2 & Grad 2 & Grad 2 & Grad 2 \\
\hline \multicolumn{2}{|l|}{ Bisherige Behandlung } & $\begin{array}{l}\text { Mastektomie mit } \\
\text { Axillaausräumung }\end{array}$ & $\begin{array}{l}\text { Mastektomie mit } \\
\text { Axillaausräumung }\end{array}$ & $\begin{array}{l}\text { SSM mit sek. } \\
\text { Axillaausräumung }\end{array}$ & $\begin{array}{l}\text { BCT mit Axillaausräumung, } \\
\text { SSM (Rezidiv) }\end{array}$ & SSM mit Axillaausräumung \\
\hline \multicolumn{2}{|l|}{ Datum Erstdiagnose } & $01 / 2008$ & $11 / 2012$ & $08 / 2014$ & 1992 09/2015 & $11 / 2013$ \\
\hline \multicolumn{2}{|l|}{ TNM-Staging } & $\begin{array}{l}\text { PT3 pN0 (0/21 LK) M0 L1 } \\
\text { G3 R1 }\end{array}$ & $\begin{array}{l}\text { PT2 pN1a (2/20 LK) M0 V0 } \\
\text { L1 G2 R0 }\end{array}$ & $\begin{array}{l}\text { yрT0 ypN1a (1/7 LK) cM0 } \\
\text { yL0 yV0 G3 R0 }\end{array}$ & $\begin{array}{l}\text { pT2 pN0 (0/8 LK) M0, } \\
\text { rpT1b pN0 (0/4) M0 L0 V0 } \\
\text { G2 R0 }\end{array}$ & $\begin{array}{l}\text { pT1c pN2 (6/10 LK) M0 V0 } \\
\text { L1 G2 R0 }\end{array}$ \\
\hline \multicolumn{2}{|l|}{ Rezeptorstatus } & $\begin{array}{l}\text { ER: IRS = } 12 \text { PR: IRS = } 4 \\
\text { HER2/neu: negativ }\end{array}$ & $\begin{array}{l}\text { ER: IRS = } 12 \text { PR: IRS = } 12 \\
\text { HER2/neu: negativ }\end{array}$ & $\begin{array}{l}\text { ER: IRS = } 6 \text { PR: IRS = } 9 \\
\text { HER2/neu: positiv }\end{array}$ & $\begin{array}{l}\text { ER: IRS = } 2 \text { PR: IRS = } 0 \\
\text { HER2/neu: negativ }\end{array}$ & $\begin{array}{l}\text { ER: IRS = } 9 \text { PR: IRS = } 6 \text { HER2 } \\
\text { neu: negativ }\end{array}$ \\
\hline \multicolumn{2}{|l|}{ Systemische Therapie } & $\begin{array}{l}\text { Adjuvant }(3 \times \text { FEC, } 3 \times \\
\text { Docetaxel), endokrine } \\
\text { Therapie: Tamoxifen }\end{array}$ & $\begin{array}{l}\text { Adjuvant }(3 \times \mathrm{TAC}) \text {, } \\
\text { endokrine Therapie: } \\
\text { Tamoxifen }\end{array}$ & $\begin{array}{l}\text { Neoadjuvant }(3 \times \text { FEC, } 3 \times \\
\text { Docetaxel) und Trastuzu- } \\
\text { mab, endokrine Therapie: } \\
\text { Tamoxifen }\end{array}$ & $\begin{array}{l}\text { Adjuvant }(4 \times \text { EC, } 12 \times \\
\text { Taxol), endokrine Therapie: } \\
\text { Tamoxifen }\end{array}$ & $\begin{array}{l}\text { Adjuvant }(4 \times \text { EC, } 12 \times \\
\text { Taxol), endokrine Therapie: } \\
\text { Tamoxifen }\end{array}$ \\
\hline \multicolumn{2}{|l|}{ Strahlentherapie } & $\begin{array}{l}\text { Brustwand und Axilla + } \\
\text { supraklavikulär }(68,4 \text { Gy) }\end{array}$ & $\begin{array}{l}\text { Brustwand und Axilla } \\
\text { (50,4 Gy) }\end{array}$ & Brustwand und Axilla & $\begin{array}{l}\text { Boost-Bestrahlung } \\
\text { Gesamtbrust }\end{array}$ & $\begin{array}{l}\text { Brustwand und Axilla + } \\
\text { supraklavikulär (50,4 Gy) }\end{array}$ \\
\hline \multicolumn{2}{|l|}{ Datum Turn-Over Flap } & $05 / 2016$ & $08 / 2016$ & $11 / 2016$ & 09/2017 & $11 / 2017$ \\
\hline \multirow{2}{*}{$\begin{array}{l}\text { Schmerzscore } \\
\text { (NASO-10) }\end{array}$} & Präop. & 8 & 5 & 8 & 2 & 7 \\
\hline & Postop. & 1 & 0 & 3 & 1 & 0 \\
\hline \multirow{2}{*}{$\begin{array}{l}\text { Behandlung } \\
\text { Lymphödem }\end{array}$} & Präop. & $\mathrm{Ja}$ & $\mathrm{Ja}$ & $\mathrm{Ja}$ & $\mathrm{Ja}$ & $\mathrm{Ja}$ \\
\hline & Postop. & Nein & Nur begrenzte Zeit & Nur begrenzte Zeit & Nein & Nein \\
\hline \multirow[t]{2}{*}{ LYMQOL-Score } & Präop. & 63 & 64 & 74 & 47 & 48 \\
\hline & Postop. & 49 & 41 & 64 & 44 & 30 \\
\hline \multirow{2}{*}{$\begin{array}{l}\text { Lebensqualität (QoL) } \\
(0=\text { sehr schlecht bis } \\
10=\text { sehr gut) }\end{array}$} & Präop. & 2 & 3 & 2 & 3 & 4 \\
\hline & Postop. & 7 & 8 & 5 & 7 & 8 \\
\hline
\end{tabular}

LYMQOL-Score: Die 4 Domänen sind: Funktion (Q1-3), Aussehen (Q4-8), Symptome (Q9-14) und Stimmung (Q15-20). Die Bewertung der einzelnen Items reicht von 1 (überhaupt nicht) bis 4 (sehr viel). BCT: brusterhaltende Therapie SSM: hautsparende Mastektomie ER: Östrogenrezeptor PR: Progesteronrezeptor 


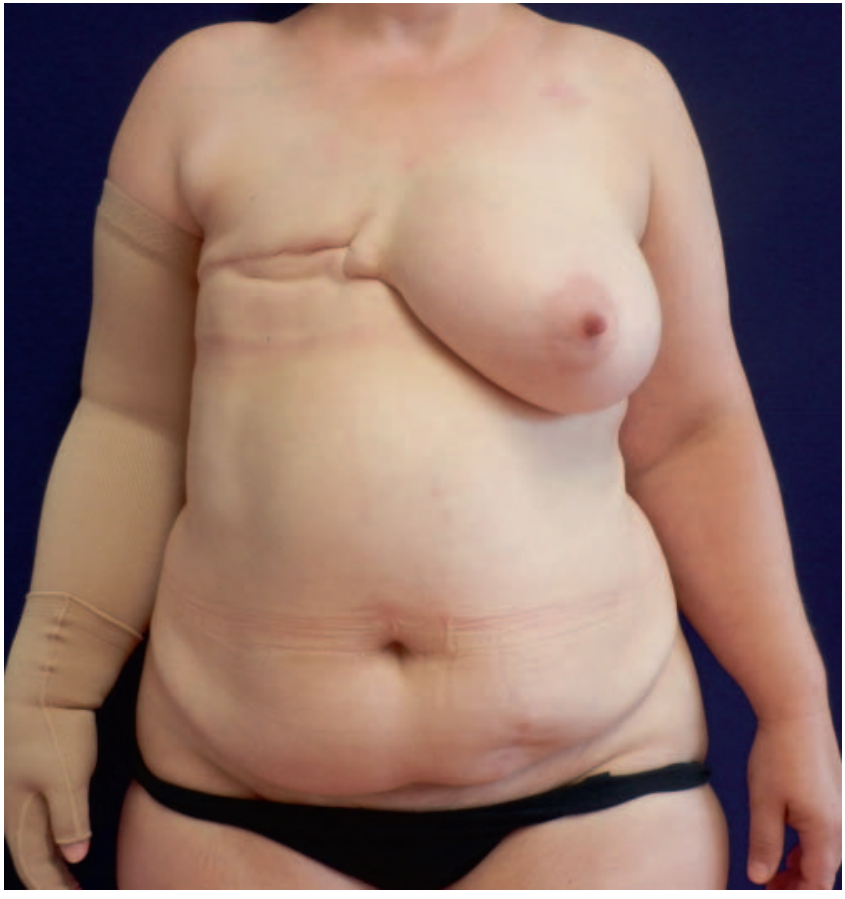

- Abb. 2 Präoperativer Situs.

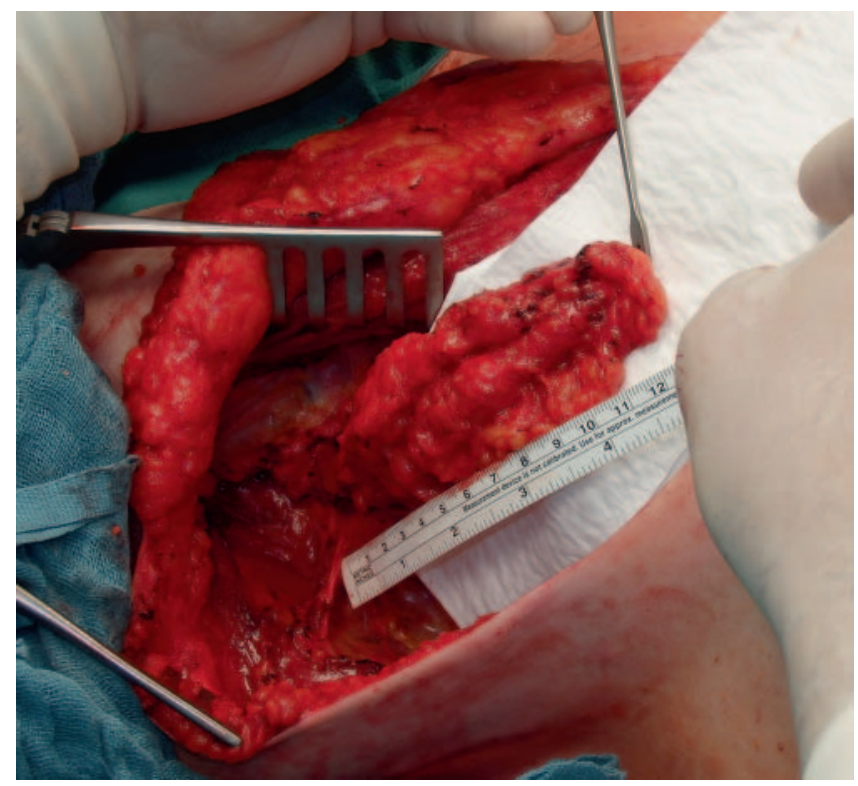

- Abb. 3 Inzidierter Wendelappen vor dem Einschwenken in die Axillarregion zur Auffüllung des Gewebedefekts nach Exzision des Narbengewebes.

\section{Diskussion}

Das Lymphödem der oberen Extremität nach Axillaausräumung mit oder ohne Strahlentherapie ist eine bekannte Komplikation bei Brustkrebspatientinnen [14]. Bei Versagen der konservativen Behandlung ist die operative Therapie eine Option für diese Patientinnen. Nach Angaben der Internationalen Gesellschaft für Lymphologie bieten operative Verfahren die größten Heilungs-

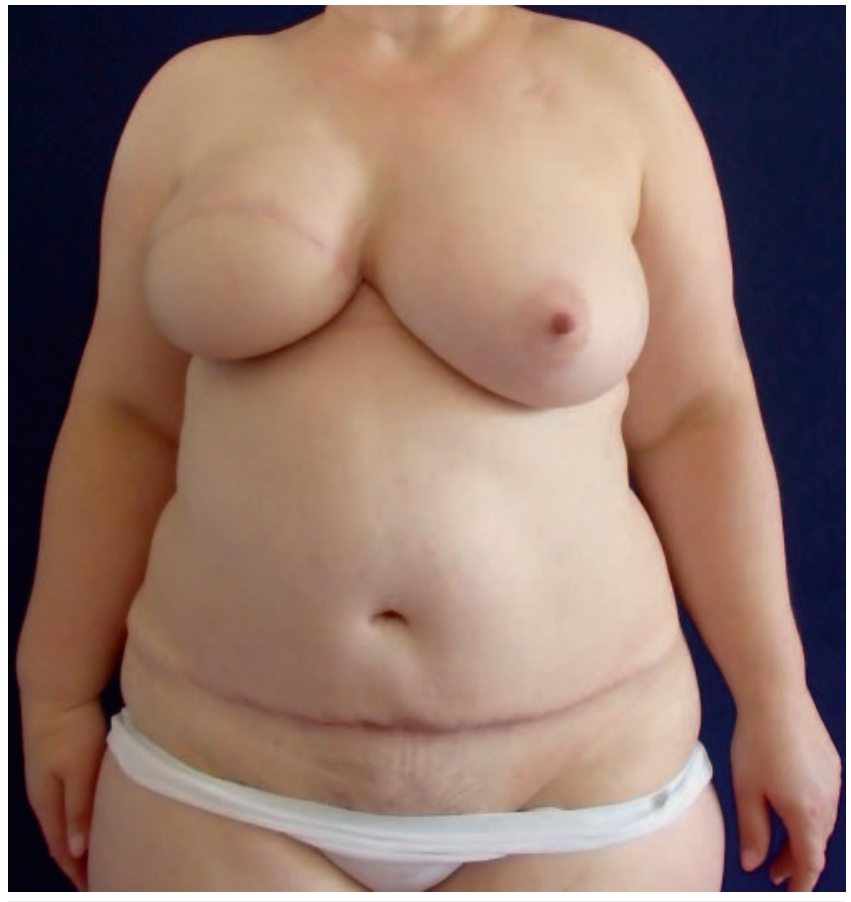

Abb. 4 OP-Situs 6 Wochen nach Eingriff.

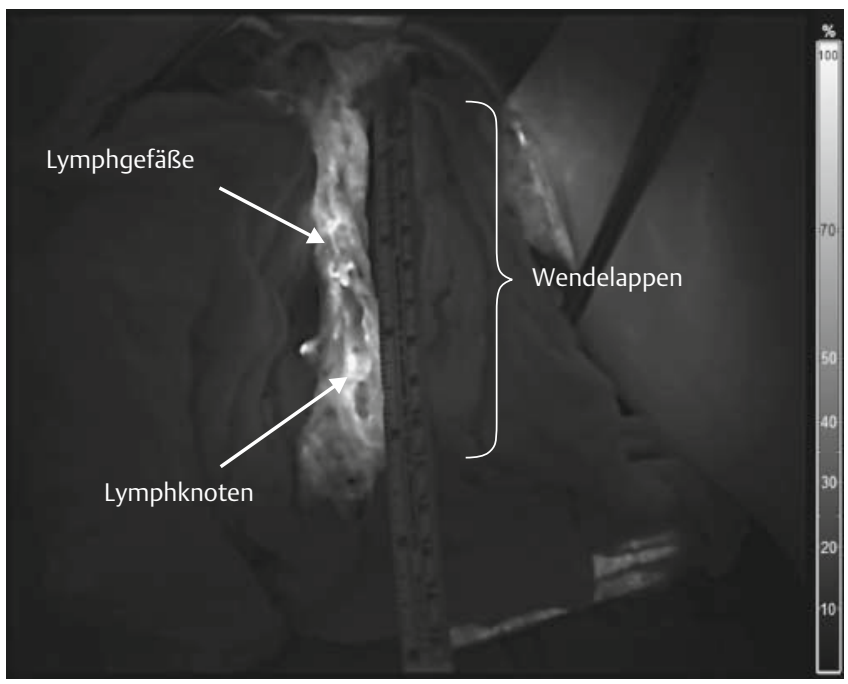

- Abb. 5 Lymphatische Perfusion nach präoperativer Injektion von Indocyaningrün bei Einsatz einer Infrarotkamera.

chancen bei Lymphabflussstörungen [15]. Alternativen mit guten Langzeitergebnissen sind die lymphatisch-venöse Anastomose (LVA) und die vaskularisierte Lymphknotentransplantation (VLNT) [16]. Häufigster Eingriff ist die LVA, die darauf abzielt, die obstruierte Region zu umgehen, indem zwischen Lymph- und Venengefäßen mehrere Anastomosen geschaffen werden, um die Lymphflüssigkeit in das Venensystem distal der Obstruktion abfließen zu lassen. Aufgrund des Druckgefälles strömt die gestaute Flüssigkeit durch die Anastomose ab. Die Wirksamkeit dieser Technik wurde nur bei Lymphödemen im Frühstadium nachgewiesen, da in fortgeschritteneren Stadien die Gewebefi- 
brose zu dysfunktionalen Lymphgefäßen führt [18]. Die vaskularisierte Lymphknotentransplantation (VLNT) ist ein weiteres mikrochirurgisches Verfahren [17]. Die häufigsten Spenderstellen sind die kontralaterale Axilla und die inguinale Lymphknotenregion. Nur wenige Studien haben versucht, neue Spenderstellen zu erschließen, die sich noch nicht bewährt haben, beispielsweise das submentale und epiploische Lymphknotengebiet sowie die Lymphknoten des großen Netzes [19-21]. Diesbezügliche Langzeitergebnisse für die VLNT fehlen. Darüber hinaus sind die Lymphknotentransplantation und die lymphatische Venenanastomose komplexe Verfahren mit einer steilen Lernkurve. Sie erfordern nicht nur mikrochirurgische Fähigkeiten, sondern auch Kenntnisse in der Lymphologie und die Fähigkeit, die entsprechenden bildgebenden Verfahren einzusetzen [15]. Eine Morbidität an der Spenderstelle ist zwar selten, kann jedoch zu schweren Komplikationen führen und schränkt die Lebensqualität weiter ein [22, 24].

Die in dieser Veröffentlichung vorgestellte Technik ist leicht zu erlernen und setzt keine umfangreichen mikrochirurgischen Kenntnisse voraus. Die Kombination aus Narbengeweberesektion und Wiederaufbau der Axilla mittels vaskularisierter Transposition von Lymphknoten und Fettgewebe der Brustwand durch das multimodale Konzept des Turn-Over Flaps stellt eine effektive und erfolgversprechende Therapieoption dar.

Wir sind der Auffassung, dass bei Brustkrebspatientinnen die zu Narbengewebe und Volumenreduktion führende Resektion des axillären Gewebes zu einer Abknickung und Obstruktion der Lymphgefäße mit sekundärer Hemmung des axillären Lymphabflusses führt. Es ist vergleichbar mit dem Abknicken eines Strohhalms, durch den keine Flüssigkeit mehr strömen kann. Durch den Wiederaufbau dieses Raums mit durchblutetem Fettgewebe und lymphatischem Gewebe aus der lateralen Thoraxwand kann der Lymphabfluss wiederhergestellt werden.

Nach unserer Erfahrung kann es bei unzureichender Resektion des Narbengewebes mit Eröffnung des Axillarraums zum Versagen der Lymphknotentransplantation kommen. Auch dann können abgeknickte Lymphgefäße als Folge von Narbenrezidiven den Lymphabfluss reduzieren.

\section{Einschränkungen}

Es handelt sich um eine retrospektive Auswertung. Die Zahl der Patientinnen ist klein. Die Aussage, dass das axilläre Narbengewebe bei Patientinnen mit Lymphödem vollständig reseziert und mit einem Wiederaufbau mittels Turn-Over Flaps kombiniert werden muss, basiert auf Beobachtungen und Erfahrungen aus mehr als 2000 Brustrekonstruktionen. Zur Untermauerung der Evidenz unserer Beobachtungen ist eine prospektive, randomisierte Studie unter standardisierten Bedingungen erforderlich. Eine weitere Frage ist, ob dieses Verfahren bei Patientinnen nach brusterhaltender Therapie und Lymphödem, die sich keiner Brustrekonstruktion unterziehen, sinnvoll wäre. Diese Patientinnen könnten auch von der Resektion des Narbengewebes in Kombination mit einem Turn-Over Flap profitieren. Daher sind weitere prospektive klinische Studien erforderlich.
Die Daten legen nahe, dass diese Operationstechnik die Behandlungsmöglichkeiten des Lymphödems der oberen Extremitäten vereinfachen würde. Die bisher vorliegenden postoperativen Ergebnisse des Turn-Over Flaps lassen vermuten, dass diese Technik keine hochspezialisierte mikrochirurgische Ausbildung erfordert und daher von vielen Mammachirurgen durchgeführt werden könnte. Darüber hinaus werden durch dieses neue Verfahren iatrogene Komorbiditäten an der Spenderstelle weitgehend vermieden.

\section{Schlussfolgerung}

Eine vollständige Entfernung der nach Brustkrebsbehandlung entstandenen störenden Vernarbungen im Bereich der Axilla und eine Wiederherstellung des Achselbereichvolumens mithilfe eines Turn-Over Flaps führten zu einer Verringerung des ipsilateralen Lymphödems und zu einer Linderung der Schmerzen und einer Steigerung der Lebensqualität. Wir deuten diesen Outcome als $\mathrm{Er}$ gebnis der physiologischen Ausrichtung der Lymphgefäße und eines lymphknotenhaltigen Transpositionslappens. Daher könnte der Turn-Over Flap eine wirkungsvolle Alternative zur StandardLymphknotentransplantation sein.

\section{Interessenkonflikt}

Die Autorinnen/Autoren geben an, dass kein Interessenkonflikt besteht.

\section{Literatur}

[1] Zou L, Liu FH, Shen PP et al. The incidence and risk factors of related lymphedema for breast cancer survivors post-operation: a 2-year follow-up prospective cohort study. Breast Cancer 2018; 25: 309-314. doi:10.1007/s12282-018-0830-3

[2] DiSipio T, Rye S, Newman B et al. Incidence of unilateral arm lymphoe dema after breast cancer: a systematic review and meta-analysis. Lancet Oncol 2013; 14: 500-515. doi:10.1016/S1470-2045(13)70076-7

[3] Taghian NR, Miller CL, Jammallo LS et al. Lymphedema following breast cancer treatment and impact on quality of life: a review. Crit Rev Oncol Hematol 2014; 92: 227-234. doi:10.1016/j.critrevonc.2014.06.004

[4] McLaughlin SA, Staley AC, Vicini F et al. Considerations for clinicians in the diagnosis, prevention, and treatment of breast cancer-related lymphedema: recommendations from a multidisciplinary expert ASBrS panel Part 1: definitions, assessments, education, and future directions. Ann Surg Oncol 2017; 24: 2818-2826. doi:10.1245/s10434-017-5982-4

[5] Tsai RJ, Dennis LK, Lynch CF et al. Lymphedema following breast cancer: The importance of surgical methods and obesity. Front Womens Health 2018. doi:10.15761/FWH.1000144

[6] Rockson SG, Rivera KK. Estimating the population burden of lymphedema. Ann N Y Acad Sci 2008; 1131: 147-154. doi:10.1196/annals. 1413.014

[7] Hayes S, Cornish B, Newman B. Comparison of methods to diagnose lymphoedema among breast cancer survivors: 6-month follow-up. Breast Cancer Res Treat 2005; 89: 221-226. doi:10.1007/s10549-004-2045-x

[8] Vieira RA, da Costa AM, de Souza JL et al. Risk factors for arm lymphedema in a cohort of breast cancer patients followed up for 10 years. Breast Care (Basel) 2016; 11: 45-50. doi:10.1159/000442489

[9] Wetzig N, Gill PG, Espinoza D et al. Sentinel-lymph-node-based management or routine axillary clearance? Five-year outcomes of the RACS Sentinel Node Biopsy Versus Axillary Clearance (SNAC) 1 trial: assessment and 
incidence of true lymphedema. Ann Surg Oncol 2017; 24: 1064-1070. doi:10.1245/s10434-016-5669-2

[10] Baumeister RG, Seifert J, Wiebecke B et al. Experimental basis and first application of clinical lymph vessel transplantation of secondary lymphedema. World J Surg 1981; 5: 401-407. doi:10.1007/BF01658013

[11] Becker C, Assouad J, Riquet M et al. Postmastectomy lymphedema: longterm results following microsurgical lymph node transplantation. Ann Surg 2006; 243: 313-315. doi:10.1097/01.sla.0000201258. 10304.16

[12] Arbeitsgemeinschaft der Wissenschaftlichen Medizinischen Fachgesellschaften e. V. (AWMF). S2k Leitlinie Diagnostik und Therapie der Lymphödeme (Mai 2017). Online (last access: 01.02.2019): https:// www.awmf.org/uploads/tx_szleitlinien/058-001I_S2k_Diagnostik_ und_Therapie_der_Lymphoedeme_2017-05.pdf

[13] Keeley V, Crooks S, Locke J et al. A quality of life measure for limb lymphoedema (LYMQOL). J Lymphoedema 2010; 5: 26-37. doi:10.1177| 0268355515586312

[14] McLaughlin SA, DeSnyder SM, Klimberg S et al. Considerations for clinicians in the diagnosis, prevention, and treatment of breast cancerrelated lymphedema: recommendations from an expert panel - Part 2: preventive and therapeutic options. Ann Surg Oncol 2017; 24: 28272835. doi:10.1245/s10434-017-5964-6

[15] International Society of Lymphology. Diagnosis and Treatment of Peripheral Lymphedema (2016). Online (last access: 01.02.2019): https://www.internationalsocietyoflymphology.org/wp-content/ uploads/2017/12/20106-35060-1-PB.pdf

[16] Baumeister RG, Mayo W, Notohamiprodjo M et al. Microsurgical lymphatic vessel transplantation. J Reconstr Microsurg 2016; 32: 34-41. doi:10.1055/s-0035-1554934
[17] Becker C. Autologous lymph node transfers. J Reconstr Microsurg 2016; 32: 28-33. doi:10.1055/s-0035-1563393

[18] Maclellan RA, Greene AK. Lymphedema. Semin Pediatr Surg 2014; 23 : 191-197. doi:10.1053/j.sempedsurg.2014.07.004

[19] Ho OA, Lin CY, Pappalardo M et al. Comparisons of Submental and Groin Vascularized Lymph Node Flaps Transfer for Breast Cancer-Related Lymphedema. Plast Reconstr Surg Glob Open 2018; 6: e1923 doi:10.1097/GOX.0000000000001923

[20] Chu YY, Allen RJ jr, Wu TJ et al. Greater Omental Lymph Node Flap for Upper Limb Lymphedema with Lymph Nodes-depleted Patient. Plast Reconstr Surg Glob Open 2017; 5: e1288 doi:10.1097/ GOX.0000000000001288

[21] Ciudad P, Manrique OJ, Date S et al. Double gastroepiploic vascularized lymph node tranfers to middle and distal limb for the treatment of lymphedema. Microsurgery 2017; 37: 771-779. doi:10.1002/micr. 30168

[22] Demiri E, Dionyssiou D, Tsimponis A et al. Donor-Site Lymphedema Following Lymph Node Transfer for Breast Cancer-Related Lymphedema: A Systematic Review of the Literature. Lymphat Res Biol 2018; 16: 2-8. doi:10.1089/Irb.2017.0043

[23] Vignes S, Blanchard M, Yannoutsos A et al. Complications of Autologous Lymph-node Transplantation for Limb Lymphoedema. Eur J Vasc Endovasc Surg 2013; 45: 516-520. doi:10.1016/j.ejvs.2012.11.026

[24] Pappalardo M, Patel K, Cheng MH. Vascularized lymph node transfer for treatment of extremity lymphedema: an overview of current controversies regarding donor sites, recipient sites and outcomes. J Surg Oncol 2018; 117: 1420-1431. doi:10.1002/jso.25034 